\title{
Thermal Cross-Section of Naturally Occurring Tantalum
}

\author{
A. K. M. Moinul Haque Meaze \\ Department of Physics, University of Chittagong, Chittagong, 4331, Bangladesh
}

\begin{abstract}
Neutron induced thermal cross-section of naturally occurring Tantalum (Ta) has been analyzed by using four different Ta samples with thickness 4.0, 2.0, 1.0 and $0.5 \mathrm{~mm}$ (respectively equivalent to $0.0224,0.0111,0.0055$, and 0.0025 atoms/barn) at the Pohang Neutron Facility, Republic of Korea. The PNF consists of a $100 \mathrm{MeV}$ electron linear accelerator (LINAC), a water cooled Tantalum (Ta) target, $12.06 \mathrm{~m}$ TOF path length, four position automatic sample changer controlled remotely by the CAMAC data acquisitions systems, and a ${ }^{6} \mathrm{LiZnS}(\mathrm{Ag})$ inorganic scintillator as a neutron detector. The distance from the water cooled Ta target to the sample changer is $5.4 \mathrm{~m}$. To reduce the gamma flash originated from the neutron target we have employed a neutron-gamma separation system based on their different pulse shape. The samples were highly pure $(\sim 99.9 \%)$. In order to estimate the background and also to calibrate the energy we used notch filter of Cobalt (Co), Indium (In), and Cadmium (Cd) that has a large resonance peaks within our analyzed energy region. The area of the each samples of notch filter was $100 \times 100 \mathrm{~mm}$, and the thickness were $0.5 \mathrm{~mm}$ for Co and Cd; and $0.2 \mathrm{~mm}$ for the In. To get more events, experiments were done around 110 hours. The unwanted events were deleted from the real events by fitting with black resonances events of notch filter. The weighted mean of the measured thermal total neutron cross-section of Ta was compared with the existing experimental as well as evaluated nuclear data files.
\end{abstract}

Keywords Thermal Cross-Sections, Electron Linear Accelerator, Neutron Detector

\section{Introduction}

Our knowledge of neutron cross-section in thermal energy zone is more complete than any other energy zone. This is because of easy getting high thermal neutron flux and concentrated of many dedicated workers. Generally there are many data of thermal cross-sections for most of the elements. However, in the case of Tantalum, unfortunately the results are few.

Tantalum is a rare, shiny, gray, dense metal which is highly corrosion resistant. It is an excellent conductor of heat and electricity. Tantalum is used in the electronics industry for capacitors and high power resistors. It is also used to make alloys to increase strength, ductility and corrosion resistance. The metal is used in dental and surgical instruments and implants, as it causes no immune response. For the neutron target it is used in different facilities.

Its high melting point and super conducting properties have made it in many metallic applications as well including filaments, wires, and acid-proof chemical equipments. It's also used to make a variety of alloys with desirable properties such as high strength, good ductility, etc. Ta also used in surgical instruments and implants because it does not react

* Corresponding author:

mhqmeaze@yahoo.com (A. K. M. Moinul Haque Meaze)

Published online at http://journal.sapub.org/jnpp

Copyright (C) 2011 Scientific \& Academic Publishing. All Rights Reserved with body fluids.

Due to these huge applications and few experimental fluctuated thermal data we measured Ta for different energy regions. We already published[1] Ta total cross-sections and resonance parameters from 0.01 to $100 \mathrm{eV}$ by using only one sample and also we showed only the data trend and hence we did not able to show exact cross-section in thermal point. Here we used four different Ta sheets in order to locate the exact thermal value.

J. R. Dunning et. al.[2] published thermal total cross-section of Ta is 27 barn in 1935. The interaction of this thermal neutron with matter was studied at Colombia University and the error of their result is around 10\%. At the Material Testing Reactor Crystal Spectrometer, USA, J. E. Evans et. al.[3] reported their thermal value is 24.3 barn in 1955. The last experimental thermal cross-section was published in 1960[ 4] and their result is almost three times higher than previous data. The nuclear data libraries[5,6] were also used different values for the reproducing of the Evaluations.

For this measurements we carried out transmission experiments on four metallic tantalum sheets with different thickness because sample thickness is of great importance in transmission measurements. Sample must be enough thick to provide a detectable amount of interactions and not too thick then the entire neutron beam interacts with the sample. As the thickness needed to provide the accurate transmissions and hence cross-sections, so we analyzed different Ta samples. Finally we have taken weighted mean value and 
compared with others.

\section{Experimental Facility}

The experiment was done at the Pohang Neutron Facility of Pohang Accelerator Laboratory, South Korea.

The PNF consists of a $100 \mathrm{MeV}$ electron linac, water-cooled Ta target and a $12 \mathrm{~m}$ long evacuated flight tube. The electron linac consists of a thermionic RF-gun, an alpha magnet, four quadruple magnets, two SLAC-type accelerating sections, a quadruple triplet and a beam-analyzing magnet. The overall length of the linac is about $15 \mathrm{~m}$. As a photo-neutron target, it is necessary to use heavy mass materials in order to produce intense neutrons by way of Bremsstrahlung under high-power electron beams. We have chosen a tantalum as the target material, which has advantages of high density $\left(16.6 \mathrm{~g}^{-\mathrm{cm}^{-3}}\right)$, high melting point $\left(3017^{\circ} \mathrm{C}\right)$ and high resistant against the corrosion by cooling water.

The TOF tubes were made by stainless steel with two different radii of 7.5 and $10 \mathrm{~cm}$. The neutron beam line was equipped with a four-position sample changer that can allow the simultaneous total cross-section measurement for four different samples.

The sample changer is a disc with four symmetrically round holes; each one is of $8 \mathrm{~cm}$ in diameter. The system rotates relatively to its own central axis and allows automatically the accumulation of data from up to four different samples in one run after setting the time exposition time individually for each sample. Thus, the sample changer provides economic and timesaving measurement. Moreover, the automatic control allows avoiding the systematic errors due to the fluctuation of the neutron beam intensity.

The four-position sample changer can be used with different samples: solid samples as well as liquid or powder ones shrouded in special cassettes. The disc cover has two control and management windows and another one for samples downloading. All of them are transparent and pressurized. The beam transmission windows - one in the fixed disk of SC, another in a top of cover are covered with aluminum foil and pressurized also. The movement of the mobile disk with samples is performed by the electric motor with the build - in reduction gearbox. The time of movement between two neighboring positions is $10 \mathrm{sec}$. The sample changer shown in Fig 1.

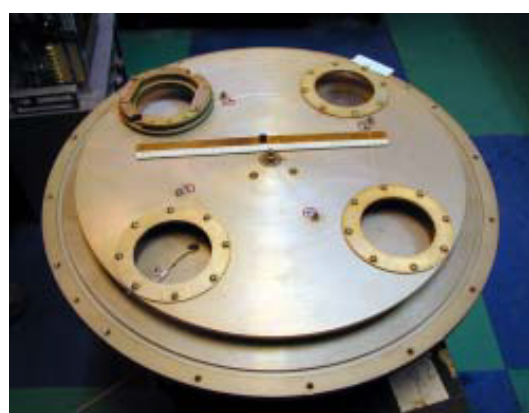

Figure 1. Four position sample changer
Pulsed neutrons were produced from a water-cooled Ta target in a water tank as a neutron moderator. The target is composed of 10 Ta plates with a radius of $2.45 \mathrm{~cm}$ and an effective thickness of $7.4 \mathrm{~cm}$. There is a $0.15 \mathrm{~cm}$ water gap between Ta plates for cooling the target effectively. The housing of the target is made by titanium. The calculated neutrons yield per $\mathrm{kW}$ of beam power at the Ta target was $2.0 \times 10^{12} \mathrm{n} / \mathrm{s}$.

The electron beam produced from the linear accelerator hit the Ta target, located in the center of a cylindrical water moderator contained in a aluminum cylinder with a thickness of $0.5 \mathrm{~cm}$, a radius of $15 \mathrm{~cm}$ and a height of $30 \mathrm{~cm}$ and the target is aligned vertically with the center of the TOF tube.

Water moderator slowed down the generated neutrons and water was $3 \mathrm{~cm}$ above the target surface. The neutron collimation system was composed of $\mathrm{H}_{3} \mathrm{BO}_{3}, \mathrm{~Pb}$, and $\mathrm{Fe}$ collimators. During the transmission measurement of $\mathrm{Ta}$ samples the linac was operated with the beam energy 65 $\mathrm{MeV}$, repetition rate $10 \mathrm{~Hz}$, and pulse width $1 \mu \mathrm{s}$.

To measure neutron TOF spectra there are three different data acquisition systems, which are NIM, CAMAC, and VME based systems.

The dynode signal from BC702 scintillator is connected though an amplifier (AMP) to a pulse shapes analyzer (PSA). A fast NIM signal from the output of PSA is delayed by $60 \mathrm{~ns}$ and is used as a start signal of the time amplitude converter (TAC). Another output signal from the PSA is used as a stop signal of the TAC. The TAC output is connected to a single channel analyzer. One of the SCA output signals is used as a stop signal of a $100 \mathrm{MHz}$ Turbo MCS (Time Digitizer) and the other is sent to a CAMAC based system. The electronics blocks used in PNF are supplied by ORTEC Company.

The CAMAC based system consists of a data acquisition part and control part of the sample changer (SC). The output signal of SCA connects to a time encoder (TE) through a detector number encoder (DNE) via G\&D Gen. Time encoder has 4096 TOF channels and a minimum dwell of 0.5 $\mu \mathrm{S}$. MEM16K is a memory of $16 \mathrm{~K}$ capacity, which collects the TOF spectra during the measurement.

The $10-\mathrm{Hz}$ RF trigger signal for the modulator of the electron linac is connected to another G\&D Gen.; one of which output is used as a start signals for both the time digitizer and the time encoder where as the other output is connected to the counter through TTL/NIM converter. TTL/NIM module is used as bilateral converter of the signals and for remote control purpose if necessary.

It can possible to send a request to change the samples for sample changer (SC) control and interrupts the measurement until the next sample reaches its position. SC blocks may operate in automatic (normal) and manual modes.

Generally NIM based system is uses to separate neutron gamma and also we may accumulate the neutron TOF spectra. We can select the different channel widths for each crate so that we may cover all the expected incident neutron energy range. And finally we can add all the energy ranges by considering the better energy resolutions for the channel 
widths of the data saving systems. However, in this analysis we only used the data of the NIM module by fixing its channel width $1.5 \mu \mathrm{s}$. By this channel width we can reach to very low energy zone.

The detector is an efficient detector of thermal neutrons in the presence of gamma radiation. The detector employs a lithium compound, whose $\mathrm{Li}$ content is enriched to $95 \%{ }^{6} \mathrm{Li}$ dispersed in a $\mathrm{ZnS}(\mathrm{Ag})$ matrix. Its design represents an improved version of that reported by Stedman. Fifty-seven efficient measurements of thermal neutron fluxes may be performed in the presence of gamma radiation as high as $10^{7}$ gamma rays per neutron.

In most applications, it will give counting efficiencies up to $100 \%$ greater than those attainable with similar detectors based on ${ }^{10} \mathrm{~B}$. The efficiency is dependent on the neutron energy and is approximately $30 \%$ for $0.1 \mathrm{eV}$ neutrons and $60 \%$ for neutrons of energy $0.01 \mathrm{eV}$ or less. The detection efficiency for thermal neutrons of about $0.025 \mathrm{eV}$ is approximately $55 \%$.

All other parameters of experiment are same as on $\operatorname{refs}[7,8]$.

\section{Data Taking and Processing}

In the measurements period the exposition times for samples Ta were 15 minutes (9000 pulses of PNF linac); for empty position, it was also 15 minutes. Thus, the duration for the sample was the same as those for the total open beam measurements. The interleaving sequence of free position of the sample changer was chosen to minimize the influence of slow and (/or) small variation of the neutron beam intensity. If the beam intensity variations or its drift was fast and (/or) large, then these partial measurements were excluded from the total statistics.

The total data taking times and Ta were 48.75 and 61.25 hrs, respectively for $4.0,0.5 \mathrm{~mm}$ and $2.0,1.0 \mathrm{~mm}$ thickness. It means we used beam on $4.0 \mathrm{~mm}$ and $0.5 \mathrm{~mm} \mathrm{Ta}$, and after that on 2.0 and $1.0 \mathrm{~mm}$ Ta in different period. We also used Notch Filter to denote black resonances, and finally to subtract background by the fitting procedure[9].

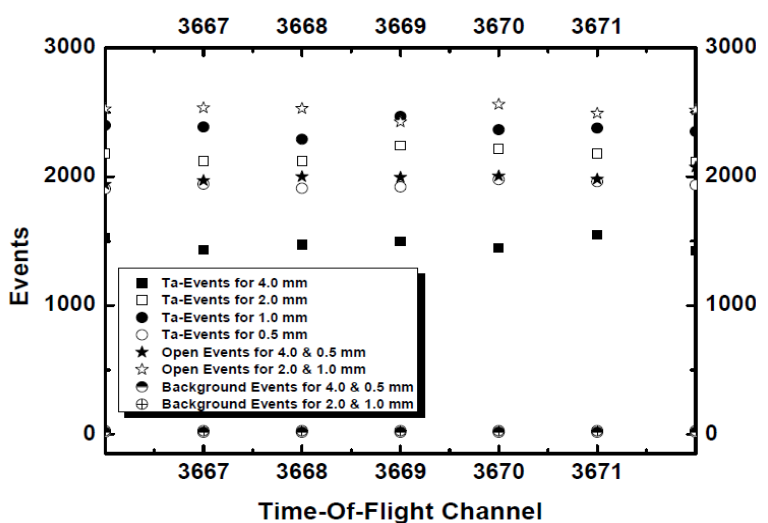

Figure 2. Tantalum events for different thickness and Open events, along-with background events located in the bottom of the figure. Due to the different experimental times, the two background levels are raised, but these are nearest and hence looked overlapping
It is noted that in the current analysis our target is to obtain thermal cross-section and so we have carefully examined only couple of time-of-flight channels by centering channel no 3669 , which is equivalent to thermal value $(0.0253 \mathrm{eV}$ or $2200 \mathrm{~m} / \mathrm{s}$ ). All the events for Ta and empty position (when there was nothing on the beam) along-with background levels are shown in Fig. 2.

We estimated the background level by using the resonance energies of the neutron TOF spectra of notch-filters of Co, In, and Cd. The magnitude of the background level was interpolated between the black resonance's by using the fitting function $y=A_{1} \exp \left(-x / t_{1}\right)+y_{0}$, where $A_{l}, t_{l}$, and $y_{0}$ are constants and $x$ is the channel number of the time digitizer.

After deleting the unwanted events we got the net events and then we have calculated the transmissions coefficient which is defined as the net ratio of the neutron counts for the Ta samples and to the open beams. As the transmission and cross-sections are energy depended, so more accurately the transmissions coefficient $(T)$ for the $j$-th group energy $E_{j}$ can be defined as the fraction of incident neutrons passing through the sample compared to that in the open beam, and its mathematical expression is :

$$
T_{r}\left(E_{j}\right)=\frac{T a\left(E_{j}\right)-B g\left(E_{j}\right)}{O p\left(E_{j}\right)-B g\left(E_{j}\right)}
$$

where $\operatorname{Ta}\left(E_{j}\right)$ and $\operatorname{Op}\left(E_{j}\right)$ are the foreground events for the Ta samples in and Open events; $\operatorname{Bg}\left(E_{j}\right)$ is the background counts for the sample in and sample out.

In this measurement, we assumed the monitor counts to be equal during the measurement.

The transmission energy dependent data for the thermal energy regions is given in Fig. 3.

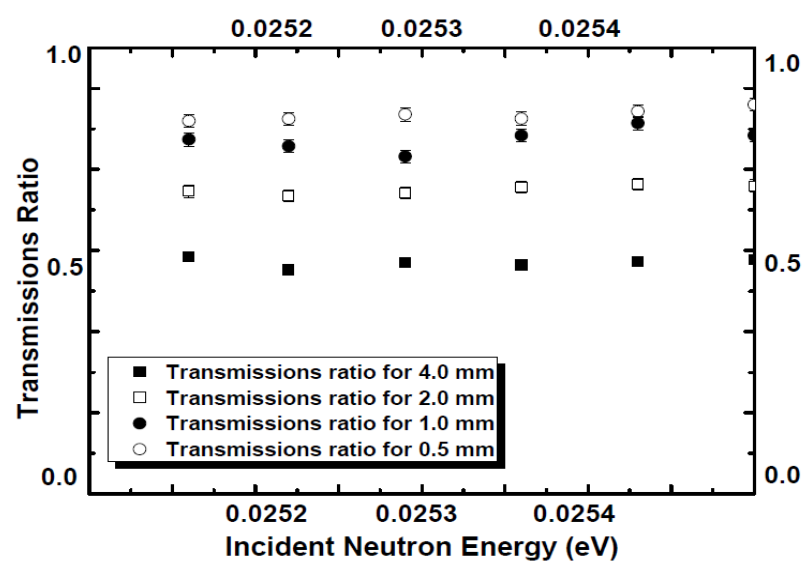

Figure 3. Transmission coefficients for the different Ta samples. It is seen that the thinner the samples, coefficients are larger

Then the energy resolution were estimated by using the equation

$$
\frac{\Delta E}{E}=2 \frac{\Delta t}{t}
$$

where the uncertainty $(\Delta t)$ of the neutron TOF (t) is composed of uncertainties due to the flight path $(2 \mathrm{~cm})$, the moderator thickness $(3 \mathrm{~cm})$, the pulse width of the electron beam $(1 \mu s)$, the channel width of the time encoder $(0.5 \mu s)$, 
and the time jitter (negligibly small) from the neutron detector.

The energy resolutions for the neutron energy of 0.01 and $0.1 \mathrm{eV}$ are respectively $0.598 \%, 0.599 \%$. This zone is the thermal energy zone and it is absolutely very good resolution for analysis.

The transmissions data in the thermal zone is also much stable so we skipped all data of the thermal zone, except exact channel number 3669 which corresponds to the 2200 $\mathrm{m} / \mathrm{s}$ or $0.0253 \mathrm{eV}$.

The neutron energy $E$ in $\mathrm{eV}$ corresponding to each time-of-flight channel $I$ in the TOF spectrum was derived from the following relation:

$$
E_{T h}=\frac{72.3 \times L}{I_{T h} \times W-\tau}
$$

where $L$ is the neutron flight path in meter, $W$ is the channel width in microseconds, and $\tau_{0}$ is the time difference between the start time from the RF trigger and the real time zero when the neutron burst was produced.

These parameters are obtained by different fitting procedures as given in ref[9].

The above transmission coefficients have been used to estimate the thermal cross-sections. The expression related to the transmission and cross-section is as follows:

$$
\sigma_{T h}=e^{-N_{T a} T_{T h}}
$$

where $\mathrm{N}_{\mathrm{Ta}}$ is the Thickness / atomic density of Ta.

\section{Results}

The thermal cross-sections for the naturally occurred Tantalum of thickness 4.0, 2.0, 1.0 and $0.5 \mathrm{~mm}$ are respectively $24.46 \pm 5.11,26.94 \pm 6.44,22.15 \pm 7.00,26.73 \pm 6.81$ (barn). The weighted mean of these results is $25.05 \pm 3.10$ (barn). This mean result along-with other available results are plotted in Fig. 4.

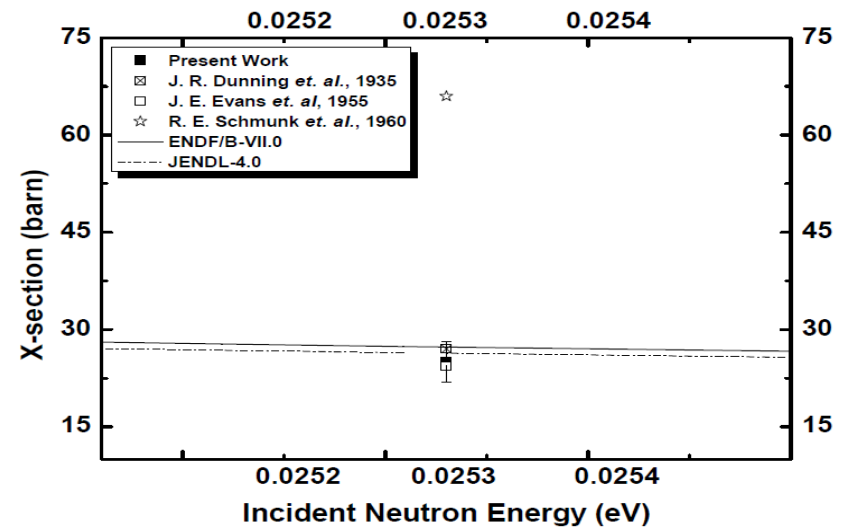

Figure 4. Thermal cross-section of Tantalum

\section{Conclusions}

The Pohang Neutron Facility (PNF) in Korea, which consists of a $100 \mathrm{MeV}$ Linac, with a Ta target, surrounded by the water moderator, and $12.06 \mathrm{~m}$ flight path has been used for the measurement of thermal total cross-sections of natural Tantalum for the incident energy $0.0253 \mathrm{eV}$.

To observe the effect of thickness we carried out transmissions experiments for four different tantalum metallic sheets with thickness $4.00,2.00,1.00$, and $0.45 \mathrm{~mm}$. The thicker the sample, the more sensitive data observed to the cross-section structure. These are more sensible to the total cross section between resonances and especially to the resonance wings. Therefore we have taken cross-section of the weighted mean of the thermal energies.

The present result is almost similar to the results of J. R. Dunning et. al.[2], J. E. Evans et. al.[3] and Japanese Evaluated Reaction Data file JENDL-4.0[6]. American Evaluate Reaction Data ENDF/B-VII.0[5] is slightly higher and R. E. Schmunk et. al.[4] data is 2.6 times higher than present result.

From the time-of-flight spectra of all Ta samples we see (in Fig. 2) that for the same time measurements for the sample of thicker thickness the number of events are higher than thinner thickness. If the thickness difference is more than the more difference is observed. Therefore, thickness is very important to get the exact cross-section. As we have used four different high purity tantalum samples and beam was carried on them for long time so the result is more reliable.

In the results section we have shown only the statistical error. The correlated error contains mainly on errors of flight path, target density, sample impurity etc which is less than $5 \%$. The total error is less than $7 \%$.

\section{ACKNOWLEDGEMENTS}

The author would like to express his sincere thanks to the staff of the Pohang Accelerator Laboratory for the excellent machine operation. The author would also like to thanks Prof. Guinyun Kim and Dr. Young Seok Lee for their wonderful cooperation and fruitful discussions.

\section{REFERENCES}

[1] A. K. M. Moinul Haque Meaze, Kunhiraman Devan, Mayeen Uddin Khandaker, Guinyun Kim, Dongchul Son, Young Seok Lee, Hengsik Kang, Moo-Hyun Cho, In Soo Ko, Won Namkung, Young-Ae Kim, Kun Joong Yoo and Young-Yuk Lee, "Measurements of Neutron Total Cross-sections of Tantalum by using the Pulsed Neutron based on an Electron Linac", Journal of the Korean Physical Society, 48 (4), 427 (2006)

[2] J. R. Dunning, G. B. Pegram, G. A. Fink and D. P. Mitchell, "Interaction of neutrons with matter", Phy Rev 48, 265 (1935)

[3] J. E. Evans E. G. Joki and R. R. Smith, "Evidence for a second naturally occurring isotopes in tantalum", Phy Rev 97, 565 (1955)

[4] R. E. Schmunk, P. D. Randolf and R.M.Brugger, "Total cross 
sections of Ti, V, Y, Ta and W", Nucl Sci Eng, 7, 195 (1960)

[5] Evaluated Nuclear Reaction Data Files, ENDF/B-VII.0, JENDL-4.0, www.nds-iaea.org

[6] Japanese Evaluated Nuclear Reaction Data Files, JENDL/4.0, JENDL-4.0, wwwndc.jaea.go.jp/jendl/j40/j40.html

[7] T. F. Wang, A. K.M. M. H. Meaze, M. U. Khandaker M. S. Rahman, G. N. Kim, L. P. Zhu, H. H. Xia, Z. Y. Zhou, Y. D. Oh, H. Kang, M. H. Cho, I. S. Ko, W. Namkung, "Measurement of the total neutron cross-section and resonance parameters of molybdenum using pulsed neutrons generated by an electron linac", Nuclear Instruments and Methods in
Physics Research, B 266, 561 (2008)

[8] AKM Moinul Haque Meaze and G N Kim, “ 209Bi(n,T) cross-sections for incident energies 0.01-100 eV", Armenian Journal of Physics, 3 (3). pp. 155-163 (2010). ISSN 1829-1171, http://ajp.asj-oa.am/280/

[9] AKM Moinul Haque Meaze and G N Kim, "Determination of niobium and palladium resonance cross-sections by timeof-flight transmission experiment", Indian Journal of Physics, Vol 85, No 35, pp. 329-338 (2011), doi: 10.1007/s12648-011-0009-7, http://www.springerlink.com/content/q7526464800r6p72/ 